\title{
The Schrödinger-Poisson equations as the large-N limit of the Newtonian N-body system: applications to the large scale dark matter dynamics
}

\author{
Fabio Briscese $\mathrm{e}^{1,2, \mathrm{a}}$ \\ ${ }^{1}$ Department of Mathematics, Physics and Electrical Engineering, Northumbria University, \\ Newcastle City Campus, Ellison Building D 207, Newcastle upon Tyne NE1 8ST, UK \\ 2 Istituto Nazionale di Alta Matematica Francesco Severi, Gruppo Nazionale di Fisica Matematica, \\ Città Universitaria, P.le A. Moro 5, 00185 Rome, Italy
}

Received: 27 March 2017 / Accepted: 7 September 2017 / Published online: 19 September 2017

(C) The Author(s) 2017. This article is an open access publication

\begin{abstract}
In this paper it is argued how the dynamics of the classical Newtonian N-body system can be described in terms of the Schrödinger-Poisson equations in the large $N$ limit. This result is based on the stochastic quantization introduced by Nelson, and on the Calogero conjecture. According to the Calogero conjecture, the emerging effective Planck constant is computed in terms of the parameters of the N-body system as $\hbar \sim M^{5 / 3} G^{1 / 2}(N /\langle\rho\rangle)^{1 / 6}$, where is $G$ the gravitational constant, $N$ and $M$ are the number and the mass of the bodies, and $\langle\rho\rangle$ is their average density. The relevance of this result in the context of large scale structure formation is discussed. In particular, this finding gives a further argument in support of the validity of the Schrödinger method as numerical double of the N-body simulations of dark matter dynamics at large cosmological scales.
\end{abstract}

\section{Introduction}

It is widely accepted tat the formation of large scale structures (LSS) in the universe, as superclusters, sheets and filaments [1-4], is shaped by collisionless dark matter (DM) [5,6] (see [7] for an historical review of DM). In the standard $\Lambda$-CDM cosmological model [8], DM is assumed to be constituted of unknown particle species that interact (almost) only gravitationally, and DM is described as a cold fluid at cosmological scales. In fact, cold dark matter (CDM) is in agreement with all cosmological data, including LSS [1-4], CMB [9,10], leansing [11], BAO [12], and supernovae [13,14] results. Even though DM particles are still elusive, there are currently many DM candidates, and the search for DM particles is an open issue [15].

\footnotetext{
a e-mail: briscese.phys@gmail.com
}

In this context, the study of the evolution of CDM becomes crucial. At large cosmological scales, CDM is successfully described as a pressureless dust fluid; but this assumption fails at smaller scales, where bound structures form. At small scales, and for realistic cases where the typical velocities are non-relativistic, the Newtonian limit of the Einstein equations is sufficient to describe the time evolution of massive bodies within the universe [16-18]. Therefore, at cosmological scales CDM can be safely described as a classical N-body system in which the individual particles represent bounded agglomerates of DM particles that interact only gravitationally.

The first and most natural way to treat this N-body system is to resort to N-body numerical simulations [19-24]. For instance, the MILLENNIUM simulation $[19,20]$ was carried out tracing the evolution of $N \sim 10^{10}$ identical particles of mass $M \simeq 10^{9} M_{\odot}$, where $M_{\odot} \simeq 2 \times 10^{30} \mathrm{~kg}$ is the solar mass. The particles in the N-body system represent huge agglomerations of elementary dark matter particles, and although in the simulation these particles do all have the same mass, this description is sufficient to explain how halos with a wide variety of masses and different abundances are built up from such effective particles. In fact, the simulation shows the formation of galactic halos made of hundreds of particles, and of clusters of galaxies made of millions of particles.

One might argue that the choice of the mass of the particles in the N-body simulation is arbitrary, and question whether this choice affects the final results. This does not seem to be the case [19-24], as far as the mass $M$ is much smaller than the mass of the objects that we study; e.g. $M$ should be much smaller than the mass of galaxies if one wants to study the formation of galactic halos, but it can be of the order of the (average) mass of galactic halos if one aims to describe the formation of LSS. Of course, a practical lower bound 
on $M$ comes from the fact that decreasing $M$, and therefore increasing the resolution attained in the N-body simulation, one increases the computational effort to solve the N-body dynamics numerically.

Thus, although the choice $M \simeq 10^{9} M_{\odot}$ in the N-body simulations is fit for purpose, it is still arbitrary. However, an indication on the plausibility of this value comes from scalar field DM models [25]. In fact, a massive and non-interacting scalar field with lagrangian density $L=\partial_{\mu} \Phi \partial^{\mu} \Phi-m_{\phi}|\Phi|^{2}$ forms bound DM halos [26] (the generalization to selfinteracting scalar fields with quartic potential has been studied in $[27,28])$. In the case of spherically symmetric scalar field configurations $\Phi=\exp \left[-i m_{\phi} t\right] \sigma(r) / \sqrt{2}$, the size of the halo is given by $l \sim \sqrt{M_{P} / \sigma(0)}\left(\hbar / m_{\phi} c\right)$. From such a relation it is quite evident that, even if $M_{P} / \sigma(0) \gg 1$ so that $l$ is much bigger than the Compton wavelength of DM particles, halos of size above $\gtrsim 10 \mathrm{kpc}$ are formed only for ultralight DM particles. The typical orbiting velocity in the halo is $v_{o} / c \sim \sqrt{\sigma(0) M_{P}}$, and using $l \sim 10 \mathrm{kpc}$ and $v_{o} \sim 100 \mathrm{~km} / \mathrm{s}$ for low luminosity spiral galaxies, one has $\sigma(0) / M_{P} \sim 10^{-6}$, while $m \sim 10^{-23} \mathrm{eV}$ [26]. We stress that this estimate of $m_{\phi}$ coincides (in order of magnitude) with that obtained in [49-52]. Furthermore, the mass $M$ of the halo in this simple model is given by $M \sim \sqrt{\sigma(0) M_{P}^{3} / m_{\phi}^{2}} \sim$ $10^{9} M_{\odot}$ [26], indeed $m \sim 10^{-23}$ is an upper bound for the DM particle mass yielding a lower bound for the masses of halos that can be realized. We mention that scalar field dark matter might be useful to resolve potential small scale problems of CDM; see [29] for an exhaustive discussion of this issue.

Due to the huge numerical effort to solve the N-body problem in realistic situations when $N \sim 10^{10}$, it would be desirable to have a simple analytical model from which it is possible to extract the most important physical properties of this N-body system. An alternative is to describe the $\mathrm{N}$-body dynamics statistically, by means of the phase-space distribution of the bodies $f(t, x, p)$, where the evolution of $f(t, x, p)$ is given by the Boltzmann equation. In the case of LSS, $\mathrm{N}$ is large and collisions are suppressed; moreover, the dynamics is only affected by the Newtonian potential [30], so that the Boltzmann equation reduces to a Vlasov (or collisionless Boltzmann) equation [31]. Although this model is simple from a conceptual point of view, there is no general solution of the Vlasov equation. However, the relevant physical information can be extracted from the momenta $M^{(n)}$ of the distribution function. To do so, one should solve the infinitely coupled hierarchy of equations for the momenta $M^{(n)}$, and it turns out that the only coherent way to neglect higher cumulants is to neglect them entirely [32]; but in this case one reduces the model to the dust model, which we know to be inappropriate to describe halo formation, while giving a good description of LSS at larger scales.
We can assume that, for our purposes, it is sufficient to study the evolution of smoothed density and velocity fields [33-35]. A possibility is to use the so called Schrödinger method (ScM), which has been proposed as numerical technique to describe the dynamics of CDM [36-52].

$\mathrm{ScM}$ is based on the hypothesis that, in the Newtonian limit, it is possible to describe the evolution of DM by means of a wave function $\psi$, such that the DM density is given by $\rho_{\mathrm{DM}}=M|\psi|^{2}$, where $M$ represents the effective mass of DM particles. The wave function $\psi$ obeys the coupled Schrödinger-Poisson equations (SPEs)

$$
\begin{aligned}
i \hbar \partial_{t} \psi & =-\frac{\hbar^{2}}{2 a(t) M} \Delta \psi+M V \psi, \\
\Delta V & =4 \pi G \rho,
\end{aligned}
$$

where $V$ is the Newtonian potential, $\rho$ is the energy density of the universe, $a(t)$ is a scale factor introduced to take into account the expansion of the universe, and $\hbar$ is a parameter representing an effective Planck constant. The Newtonian potential $V$ is determined through the Poisson equation in (1). The form of the DM density used to run cosmological simulations in [36-52] is $\rho=\left(M|\psi|^{2}-\rho_{\text {crit }}\right) / a(t)$, where $\rho_{\text {crit }}$ is a parameter representing a comoving critical density of the universe, although some authors assume $\rho_{\text {crit }}=0$, so that the SPEs reduce to the Schrödinger-Newton equations [53]. However, since we are not interested in discussing the explicit form of the DM density, we will use the generic expression $\rho$. Furthermore, here we are only focused on the relation between the $\mathrm{ScM}$ and $\mathrm{N}$-body simulations, and this is not related to the expansion of the universe; thus, hereafter we set $a(t)=1$.

The SPEs can be viewed as the non-relativistic limit of the Klein-Gordon and Dirac equations, and their theoretical justification follows from the correspondence principle that relates classical and quantum mechanical phase-spacedistribution functions in the semiclassical limit [54]. In this case $\hbar$ coincides with the Planck constant, and $M$ is the mass of the elementary DM particles. Numerical solutions of the SPEs point towards ultralight DM particles of mass $M \sim 10^{-23} \mathrm{eV}$ [49-51].

Alternatively, the $\mathrm{ScM}$ has been introduced as a numerical double of the N-body description of DM at large scales; see e.g. $[36,37,48]$ and the references therein. In this case, the effective particles in the $\mathrm{N}$-body system are again huge agglomerates of DM elementary particles, and therefore $M$ is huge in comparison with the mass of elementary DM particles. Moreover, the constant $\hbar$ in (1) does not coincide with the Planck constant, nor is fixed by the N-body problem, but it is merely a free parameter that can be chosen at will. Furthermore, due to the correspondence principle [54], $\hbar$ determines the phase-space resolution in the ScM. To ensure the match with $\mathrm{N}$-body simulations one must require 
$\hbar / M \sim 10^{-4} \mathrm{Mpc} \cdot \mathrm{c}$, so that $\hbar$ is huge in comparison with the true Planck constant.

It is worth to emphasize the difficulty in reconciliating such huge values of $M$ and $\hbar$ with the derivation of the SPEs from the fundamental quantum mechanical evolution of DM particles. In fact, even though one might assume that $M$ represents the mass of huge agglomerates of DM particles, one encounters the insurmountable problem of explaining the extremely large value of $\hbar$. In fact, since the Planck constant is fundamental, its value should not be affected by the Newtonian and semiclassical limits.

In this paper we discuss the relation between the $\mathrm{ScM}$ and the $\mathrm{N}$-body description of DM used in numerical simulations [19-24], and we argue how the SPEs can be obtained as the large $N$ limit of the Newtonian N-body system. This argument is valid beyond the context of LSS formation, and it implies that any Newtonian $\mathrm{N}$-body system of identical bodies can be described by means of the SPEs, and that makes this finding of wide interest. For completeness, we mention that the correspondence between the ScM method and the Vlasov equation has been extensively studied, and we remind the interested reader of the existing literature; see [48] and the references therein.

Our staring point is the Newtonian N-body system of DM agglomerates considered in [19-24]. We show that the dynamics of this system satisfies the hypothesis of the so called Nelson stochastic quantization [55] in the large N limit. That implies that the evolution of the system can be described statistically, by means of the Schrödinger equation. The stochastic background responsible for the Nelson quantization is given, as in the Calogero conjecture, by the gravitational interaction between the $\mathrm{N}$ bodies, and its stochastic character is due to the chaotic behavior of the N-body dynamics. What is more, the Calogero conjecture also allows one to estimate the order of magnitude of the effective Planck constant.

To begin, let us discuss briefly the hypothesis of the Nelson stochastic quantization [56], and let us consider a particle of mass $M$ which moves according to the Newton laws of motion. The further assumption is that this particle constantly undergoes a Brownian motion with no friction, and with a diffusion coefficient $\hbar / M$ inversely proportional to its mass $M$. Therefore, the trajectory of this particle will be given by

$M \ddot{\boldsymbol{x}}=-\nabla \phi+\boldsymbol{B}(t)$

where $-\nabla \phi$ represents all the conservative forces, and $\boldsymbol{B}(t)$ is a random variable with zero mean, representing a small random noise. Nelson has shown [55] that, under these hypotheses, the motion of the particle can be described by means of a stochastic process, and the probability distribution $f(x)$ of the particle can be expressed as $f(x)=|\psi|^{2}$, in terms of a wave function $\psi$ satisfying the Schrödinger equation $i \hbar \partial_{t} \psi=-\frac{\hbar^{2}}{2 M} \Delta \psi+\phi \psi$

Therefore, in this picture the quantum behavior of the dynamics of the particle is not a fundamental property of the nature, but it is induced by the random field $B(t)$. It is necessary to note that the Nelson quantization only implies the emergence of the Schrödinger equation (3), which of course, does not encompass all the features of quantum mechanics. For instance, all the properties related to the measurement processes in quantum mechanics, e.g. entanglement, have not been derived in the context of Nelson quantization. However, we are not interested in discussing the validity of the Nelson quantization as a real theory of the quantum world, and we refer the reader interested in this problem to the literature (see [56] and the references therein); but we want to exploit the result of Nelson in the context of N-body dynamics.

At this point, one can ask the question of the nature of the random field $B(t)$ responsible for the emergent quantization. One of the most studied possibilities is that $B(t)$ is the random zero-point radiation field of the electromagnetic field [56]. Another possibility, conjectured by Calogero [57,58], is that the random noise $B(t)$ is the resultant of the gravitational interaction of the particle with all the other particles of the universe. In fact, apart from the interaction with neighboring bodies which is not small and must be included in the potential $\phi$, the gravitational interaction with far bodies behaves as a small background noise, and its random behavior comes from the fact that the classical dynamics of a N-body system is chaotic (see for instance [59] for a review of classical chaos). In the context of cosmological simulations, it has been shown that chaos appears at scales smaller than a critical transition scale $\sim 3.5 \mathrm{Mpc} / \mathrm{h}$, where $h$ is the dimensionless Hubble parameter, while the dynamics appears to be nonsensitive to initial conditions (thus non-chaotic) at larger scales; see [60] for more details.

Therefore, any particle of the N-body system experiences a stochastic gravitational acceleration due to the rest of the system. Exploiting this idea, Calogero has shown that the order of magnitude of the induced Planck constant is [57]

$\hbar \sim M^{5 / 3} G^{1 / 2}(N /\langle\rho\rangle)^{1 / 6}$,

where $N$ and $M$ are the number and the mass of the bodies, $\langle\rho\rangle$ is the average density of the system, and $G$ the gravitational constant.

Let us briefly describe how (4) can be obtained on the basis of semiquantitative arguments. The relevant quantities in our analysis are the dimensional parameters $G, M$, and $\langle\rho\rangle$; plus $N$, which of course is dimensionless. From these quantities we can define the unit of time $T$ as

$T \sim(G\langle\rho\rangle)^{-1 / 2}$. 
We want to estimate the characteristic time $\tau$ of the stochastic acceleration that each particle of the system undergoes due to all the other particles. Since the N-body dynamics is chaotic, it is plausible that the characteristic frequency of this motion $\nu \sim 2 \pi / \tau$ should be a growing function of $N$, and since the background gravitational noise is due to a collective stochastic effect, it is also plausible to assume that $v$ is proportional to the square root of $N$, so that

$\tau \sim N^{-1 / 2} T$

The "quantum" of action (that is, the characteristic action) associated with the stochastic gravitational noise is obtained multiplying $\tau$ by the gravitational energy per particle $\epsilon$, which is estimated as

$\epsilon \sim G(N M)^{2} R^{-1} N^{-1} \sim G M^{5 / 3} N^{2 / 3}\langle\rho\rangle^{-1 / 3}$,

where the length $R \equiv(N M /\langle\rho\rangle)^{1 / 3}$ represents the average linear size of the N-body system. Therefore, the effective Planck constant is obtained: $\hbar \equiv \epsilon \tau$, which finally gives Eq. (4). At that point we should emphasize that this argument is not a pure dimensional analysis since, even though the exponents of the dimensional quantities in (4) are fixed by their dimensions, the dependence on the dimensionless quantity $N$ is fixed by the assumption made in (6), which plays a fundamental role in the derivation of (4). We stress that (6) can be justified in a more rigorous way, and we refer the reader to $[57,58]$, where this relation has been derived through a more detailed analysis of the properties of the Newtonian N-body system.

Let us come back to our gravitational N-body system. Using the Calogero conjecture, we have argued that, due to the classical gravitational interaction with all the other particles, any particle in the system undergoes a stochastic gravitational noise which plays the role of the stochastic random noise $B(t)$ in (2). Thus, the dynamics of each particle of the system is given in terms of a wavefunction $\psi$ solution of (3), where $\hbar$ is given by (4). At that point, we can express the wavefunction of the entire system using the Hartree-Fock approximation, so that the number density of the N-body system will be $n(x)=N|\psi|^{2}$. This is the ana$\log$ of the derivation of the famous Gross-Pitaevskii equation [62] for a Bose-Einstein condensate by means of the Hartree-Fock approximation; see [61] for a detailed analysis of the quantum many-body system of bosons. Finally, the potential in (3) is $\phi=M V$, where $V$ is the gravitational potential solution of the Poisson equation, $\Delta V=4 \pi G \rho$, where $\rho=\operatorname{Mn}(x)$.

Of course, this analysis is accurate only for $N$ large, and therefore we conclude the dynamics of the N-body system is well described by the SPEs (1) in the large N limit. Finally, we stress that the resolution of the SPEs in the phase space is fixed by $\hbar$, which in turn is fixed by the corresponding N-body problem. However, for given values of $\langle\rho\rangle$ and $N$, this resolution is improved on decreasing $M$, which is also true for the corresponding N-body system.

We can now exploit this result in the context of the LSS formation. In fact, if we come back to the description of the CDM dynamics at large scales as a Newtonian system of $N \sim 10^{10}$ bodies (which in our case represent huge aggregations of DM particles) of mass $M$, which interact only gravitationally, we immediately realize that the hypothesis of the Nelson quantization are satisfied, as in the Calogero conjecture. In this picture, the gravitational interaction produces the background random field $\boldsymbol{B}(t)$, which in turn induces the Nelson quantization, and this fact justifies the quantum mechanical treatment of the system by means of the SPEs (1).

The advantage of this deduction of SPEs from the N-body dynamics is that we can use the Calogero result (4) to estimate the order of magnitude of $\hbar$ in terms of the parameters of the $\mathrm{N}$-body problem, so that $\hbar$ is no longer a free parameter. In a virialized system of size $L$ with velocity dispersion $\sigma$, the resolution in phase space in a Schrödinger code is given by $\Delta x \Delta v \sim \sigma L / N_{G}$, where $N_{G}=L / d$ is the number of grid points and $d$ is the grid spacing in the simulation $[36,37]$. This estimate must be compared with the value $\Delta x \Delta v \sim \hbar / M \sim$ $M^{2 / 3} G^{1 / 2}(N /\langle\rho\rangle)^{1 / 6}$ obtained from (4). For instance, in the case of the MILLENNIUM simulation [19,20], where the Nbody problem is solved for $N \simeq 10^{10}$ particles of mass $M \simeq$ $10^{9} M_{\odot}$, using $\langle\rho\rangle \simeq 3 H_{0}^{2} / 8 \pi G \simeq 4 \times 10^{-26} \mathrm{~kg} / \mathrm{m}^{3}$, where $H_{0} \simeq h^{-1} \times 100 \times \mathrm{km} / \mathrm{s} \mathrm{Mpc}$, with $h \simeq 0.73$, is the Hubble constant, one has $\hbar \simeq 2 \times 10^{66} \mathrm{~kg} \mathrm{~m}^{2} / \mathrm{s}$. This corresponds to a value $\hbar / M \simeq 10^{-4} \mathrm{Mpc} \cdot \mathrm{c}$ in the range of values used in numerical simulations, e.g. $\hbar / M \sim 10^{-4} \mathrm{Mpc} \cdot \mathrm{c}$ in $[48,50]$ or $\hbar / M \sim 10^{-6} \mathrm{Mpc} \cdot \mathrm{c}$ in $[49,51]$.

In conclusion, in this paper it has been shown that the dynamics of the classical Newtonian N-body system is well described in terms of the SPEs in the large $N$ limit. This is due to the stochastic quantization of the N-body system induced by the random gravitational background produced by the $\mathrm{N}$ bodies, as in the Calogero conjecture. Moreover, the emerging effective Planck constant in the SPEs can be computed by means of (4) in terms of the parameters of the corresponding N-body system.

When applied to LSS formation, this finding gives a further argument in support of the validity of the Schrödinger method as numerical double of the N-body simulations of DM dynamics at large cosmological scales [19-24], and it offers a natural justification for the huge value of $\hbar$ often used in numerical solutions of SPEs. These results are particularly remarkable, since this derivation of SPEs in the context of the Schrödinger method is the first practical application of the Nelson quantization and of the Calogero conjecture to a realistic physical problem. 
During the proofreading of this manuscript, the author has noticed a paper [63], where it has been presented a generalized Schrödinger equation derived from the theory of scale relativity, and its application to the problem of dark matter halos formation has been discussed. Due to the links to the results presented in this manuscript, such paper has been included in the literature.

Acknowledgements The author is very grateful to F. Calogero for useful discussions on the Calogero conjecture, and to G. Rigopopulos and I. Moss for useful discussions on the draft version of this paper.

Open Access This article is distributed under the terms of the Creative Commons Attribution 4.0 International License (http://creativecomm ons.org/licenses/by/4.0/), which permits unrestricted use, distribution, and reproduction in any medium, provided you give appropriate credit to the original author(s) and the source, provide a link to the Creative Commons license, and indicate if changes were made. Funded by SCOAP ${ }^{3}$.

\section{References}

1. V. Springel et al., Nature (London) 435, 629 (2005). arXiv:astro-ph/0504097

2. E. Tempel et al., Mon. Not. R. Astron. Soc. 438, 3465 (2014). arXiv:1308.2533 [astro-ph.CO]

3. M. Tegmark et al., SDSS Collaboration, Phys. Rev. D 69, 103501 (2004). arXiv:astro-ph/0310723

4. U. Seljak et al., SDSS Collaboration, Phys. Rev. D 71, 103515 (2005). arXiv:astro-ph/0407372

5. T.J. Sumner, Living Rev. Relativ. 5, 4 (2002)

6. G. Bertone, D. Hooper, J. Silk, Phys. Rep. 405, 279-390 (2005)

7. G. Bertone, D. Hooper, arXiv:1605.04909 [astro-ph.CO]

8. V. Mukhanov, Physical Foundations of Cosmology (Cambridge University Press, Cambridge, 2005)

9. Planck Collaboration, P.A.R. Ade et al., Planck 2015 results, XIII, Cosmological Parameters. A\&A 594, A13 (2016). arXiv:1502.01589. doi:10.1051/0004-6361/201525830

10. Planck Collaboration, P.A.R. Ade et al., Planck 2015, XX, Constraints on Inflation. arXiv:1502.02114

11. B. Jain, A. Taylor, Phys. Rev. Lett. 91, 141302 (2003). arXiv:astro-ph/0306046

12. D.J. Eisenstein et al., SDSS Collaboration, Astrophys. J. 633, 560 (2005). arXiv:astro-ph/0501171

13. S. Perlmutter et al., SNCP Collaboration, Astrophys. J. 517, 565 (1999). arXiv:astro-ph/9812133

14. A.G. Riess et al., Supernova Search Team Collaboration, Astron. J. 116, 1009 (1998). arXiv:astro-ph/9805201

15. F.S. Queiroz, arXiv:1605.08788 [hep-ph]

16. N.E. Chisari, M. Zaldarriaga, Phys. Rev. D 83, 123505 (2011). arXiv:1101.3555 [astro-ph.CO]

17. S.R. Green, R.M. Wald, Phys. Rev. D 85, 063512 (2012). arXiv:1111.2997 [gr-qc]

18. M. Kopp, C. Uhlemann, T. Haugg, J. Cosmol. Astropart. Phys. 3, 018 (2014). arXiv:1312.3638 [astro-ph.CO]

19. V. Springel et al., Nature 435, 629 (2005)

20. V. Springel, C.S. Frenk, S.D.M. White, Nature (London) 440, 1137 (2006). arXiv:astro-ph/0604561

21. R. Teyssier, Astron. Astrophys. 385, 337 (2002). arXiv:astroph/0111367

22. M. Boylan-Kolchin et al., Mon. Not. R. Astron. Soc. 398, 1150 (2009). arXiv:0903.3041 [astro-ph.CO]
23. T. Abel, O. Hahn, R. Kaehler, Mon. Not. R. Astron. Soc. 427, 61 (2012). arXiv:1111.3944 [astro-ph.CO]

24. O. Hahn, T. Abel, R. Kaehler, Mon. Not. R. Astron. Soc. 434, 1171 (2013). arXiv:1210.6652 [astro-ph.CO]

25. A. Suarez, V.H. Robles, T. Matos, Astrophys. Sp. Sci. Proc. 38 , 107-142 (2014). arXiv:1302.0903 [astro-ph.CO]

26. A. Arbey, J. Lesgourgues, P. Salati, Phys. Rev. D 64, 123528 (2001). arXiv:astro-ph/0105564

27. A. Arbey, J. Lesgourgues, P. Salati, Phys. Rev. D 65, 083514 (2002). arXiv:astro-ph/0112324

28. F. Briscese, Phys. Lett. B 696, 315-320 (2011). arXiv:1101.0028 [astro-ph.CO]

29. L. Hui, J.P. Ostriker, S. Tremaine, E. Witten, Phys. Rev. D 95, 043541 (2017). arXiv:1610.08297 [astro-ph.CO]

30. I.H. Gilbert, Astrophys. J. 152, 1043 (1968)

31. H. Andreasson, Living Rev. Relativ. 14, 4 (2011)

32. S. Pueblas, R. Scoccimarro, Phys. Rev. D 80, 043504 (2009). arXiv:0809.4606 [astro-ph]

33. A. Dominguez, Phys. Rev. D 62, 103501 (2000)

34. T. Buchert, A. Domynguez, Astron. Astrophys. 438, 443 (2005). arXiv:astro-ph/0502318

35. M. Pietroni, G. Mangano, N. Saviano, M. Viel, J. Cosmol. Astropart. Phys. 1, 019 (2012). arXiv:1108.5203 [astro-ph.CO]

36. L.M. Widrow, N. Kaiser, Astrophys. J. 416, L71-L74 (1993)

37. G. Davies, L.M. Widrow. arXiv:astro-ph/9607133

38. P. Coles, K. Spencer, Mon. Not. Roy. Astron. Soc. 342, 176 (2003)

39. C.J. Short, P. Coles, J. Cosmol. Astropart. Phys. 12, 012 (2006)

40. D. Giulini, A. Groardt, Class. Quant. Grav. 29, 215010 (2012)

41. E.R. Arriola, J. Soler, J. Stat. Phys. 103, 1069 (2001)

42. I.M. Moroz, R. Penrose, P. Tod, Class. Quant. Grav. 15, 2733 (1998)

43. R. Johnston, A.N. Lasenby, M.P. Hobson, arXiv:0904.0611 [astroph.CO]

44. I. Szapudi, N. Kaiser, Astrophys. J. Lett. 583, L1 (2003)

45. M. Schaller et al., Mon. Not. R. Astron. Soc. 442(4), 3073-3095 (2014). arXiv:1310.5102 [astro-ph.CO]

46. H.-Y. Schive, T. Chiueh, T. Broadhurst, Nat. Phys. 10, 496-499 (2014)

47. H.-Y. Schive, T. Chiueh, T. Broadhurst, Astrophys. J. 818(1), 89 (2016)

48. C. Uhlemann, M. Kopp, T. Haugg, Phys. Rev. D 90, 023517 (2014)

49. J. Zhang, Y.-L.S. Tsai, K. Cheung, M.-C. Chu, arXiv:1611.00892

50. A. Paredes, H. Michinel, Phys. Dark Univ. 12, 50-55 (2016)

51. H.-Y. Schive, T. Chiueh, T. Broadhurst, Nat. Phys. 10, 496-499 (2014)

52. L.A. Urena-Lopez, Phys. Rev. D 90(2), 027306 (2014). arXiv:1310.8601 [astro-ph.CO]

53. R. Ruffini, S. Bonazzola, B. Silvano, Phys. Rev. 187(5), 1767-1783 (1969)

54. K. Takahashi, Prog. Theor. Phys. Suppl. N0, 98 (1989)

55. E. Nelson, Phys. Rev. 150, 4 (1966)

56. L. de la Peña, A.M. Cetto, A. Valdes Hernandez, The Emerging Quantum (Springer, Berlin, 2015)

57. F. Calogero, Phys Lett. A 228, 335-346 (1997)

58. F. Calogero, Int. J. Mod. Phys. B 18(4, 5), 519-525 (2004)

59. S.H. Strogatz, Nonlinear Dynamics and Chaos: With Applications to Physics, Biology, Chemistry and Engineering (Studies in Nonlinearity), 2nd edn. (Westview Press, New York, 2015)

60. J. Thiebaut, C. Pichon, T. Sousbie, S. Prunet, D. Pogosyan, Mon. Not. R. Astron. Soc. 387(1), 397-406 (2008). arXiv:0803.3120 [astro-ph]

61. Lev Pitaevskii, Sandro Stringari, Bose-Einstein Condensation (Clarendon Press, Oxford, 2003)

62. F. Dalfovo, S. Giorgini, L.P. Pitaevskii, S. Stringari, Rev. Mod. Phys. 71, 463 (1999)

63. P-H. Chavanis, Eur. Phys. J. Plus 132(6), 286 (2017). arXiv: 1706.05900 [gr-qc] 